Cerebrovasc Dis 2012;34:326-327

DOI: $10.1159 / 000342759$

\section{FLAIR with Contrast Linked to Better Correlation with Stroke Symptoms than Diffision-Weighted Imaging in a Patient: Detecting Hyperintense Acute Reperfusion Injury Marker and Cortical Enhancement}

Elissaios Karageorgiou ${ }^{\mathrm{a}, \mathrm{b}}$, Daniel J. Schwabc,

a Department of Neurology, University of Minnesota, ${ }^{b}$ Brain Sciences Center, Veterans Affairs Medical Center, and 'Department of Radiology, University of Minnesota, Minneapolis, Minn., USA

\section{Background}

Blood-brain barrier (BBB) and blood-CSF barrier (BCSF) disruption (BBBD and BCSFD) is observed in several forms of brain injury [1], including acute ischemic stroke [2, 3], hemorrhagic transformation [2], intracerebral hemorrhage [4], and carotid artery stenting [5]. This is rarely evaluated in clinical practice, while its contribution to total brain injury and its clinical significance are unclear. Fluid-attenuated inversion recovery (FLAIR) with contrast (FWC) sequences are sensitive in identifying BBBD [2]. Gadolinium enhancement at the BBB and BCSF border in FWC sequences of patients with vascular events has been coined 'hyperintense acute reperfusion injury marker' (HARM) [4]. HARM is usually noted over the sulcal subarachnoid space adjacent to lesioned brain parenchyma, on average $12.9 \mathrm{~h}$ from stroke onset, and its pathology is considered different from the leptomeningeal and parenchymal enhancement noted days to weeks after a stroke $[2,3]$. However, its independent contribution to symptomatic deficits is unverified. Through this case report we highlight the utility of FWC in acute stroke diagnosis, especially when clinical symptoms are dissociated from other MRI results.

\section{Case Report}

An 81-year-old right-handed male presented with complaints of 'not tracking and gait difficulties'. According to his daughter, he had been 'inattentive to the left' for the previous 3 days, while the patient denied any changes to his health. Examination revealed visual and somatosensory neglect on the left and executive deficits. Suspected lesion localization included the right parietal lobe, given inattention and anosognosia, and the frontal lobe, given executive dysfunction. An MRI stroke protocol was obtained (fig. 1a-c). Given the lack of a prominent parietal lesion to explain the patient's inattention, an FWC sequence was added (fig. 1d) to evaluate for BBBD and BCSFD revealing an extensive area of HARM over the right parietotemporal cortex, subregions of which also had cortical enhancement. The above supported a diagnosis of watershed stroke over the right middle cerebral artery distribution with subsequent right parietotemporal cortex reperfusion injury.

\section{Discussion}

HARM is a little-known imaging marker for the evaluation of acute stroke which has been identified in $33 \%$ of ischemic stroke patients [2], 85\% of intracerebral hemorrhage patients [4], and $57 \%$ of carotid artery stenting patients [5]. HARM can help in ischemic stroke prognosis since it has been observed in $73 \%$ of patients with subsequent hemorrhagic transformation versus only $25 \%$ of patients without hemorrhagic transformation [2]. HARM may be distant from the intraparenchymal lesion $[3,4]$ and is more frequently noted after thrombolysis [6]. Theories on HARM pathophysiology focus on matrix metalloproteinase involvement in microvasculature damage, where endogenous tissue plasminogen activator release during vascular injury increases matrix metalloproteinases 2 and 9 [1, 7]. Thus, inclusion of FWC in MRI stroke protocols can help identify an additional pathophysiological component in the setting of reperfusion injury. Finally, the literature to date has not correlated HARM with clinical symptoms independent of the underlying intraparenchymal pathology. The current case report demonstrates that HARM in FWC is associated with clinical deficits not explained by other MRI sequences. This raises the possibility that transient ischemic attacks and imaging-negative strokes might be HARM positive, and linked to focal BBBD and BCSFD.

\section{Disclosure Statement}

The authors have nothing to disclose.

\section{References}

1 Rosenberg GA: Neurological diseases in relation to the blood-brain barrier. J Cereb Blood Flow Metab 2012;32:1139-1151.

2 Latour LL, Kang DW, Ezzeddine MA, Chalela JA, Warach S: Early blood-brain barrier disruption in human focal brain ischemia. Ann Neurol 2004;56:468-477.

- 3 Dechambre SD, Duprez T, Grandin CB, Lecouvet FE, Peeters A, Cosnard G: High signal in cerebrospinal fluid mimicking subarachnoid haemorrhage on FLAIR following acute stroke and intravenous contrast medium. Neuroradiology 2000;42:608-611.

-4 Kidwell CS, Burgess R, Menon R, Warach S, Latour LL: Hyperacute injury marker (HARM) in primary hemorrhage: a distinct form of CNS barrier disruption. Neurology 2011;77:1725-1728.

5 Ogami R, Nakahara T, Hamasaki O, Araki H, Kurisu K: Cerebrospinal fluid enhancement on fluid attenuated inversion recovery images after carotid artery stenting with neuroprotective balloon occlusions: hemodynamic instability and blood-brain barrier disruption. Cardiovasc Intervent Radiol 2011;34:936-941.

6 Warach S, Latour LL: Evidence of reperfusion injury, exacerbated by thrombolytic therapy, in human focal brain ischemia using a novel imaging marker of early blood-brain barrier disruption. Stroke 2004;35: 2659-2661.

7 Batra A, Latour LL, Ruetzler CA, Hallenbeck JM, Spatz M, Warach S, Henning EC: Increased plasma and tissue MMP levels are associated with BCSFB and BBB disruption evident on post-contrast FLAIR after experimental stroke. J Cereb Blood Flow Metab 2010;30:1188-1199.

\section{KARGER}

Fax +41613061234 E-Mail karger@karger.ch www.karger.com (c) 2012 S. Karger AG, Basel

$1015-9770 / 12 / 0344-0326 \$ 38.00 / 0$

Accessible online at: www.karger.com/ced
Elissaios Karageorgiou, MD

Department of Neurology, University of Minnesota

MMC 295, 420 Delaware SE

Minneapolis, MN 55455 (USA)

E-Mail karag001@umn.edu 


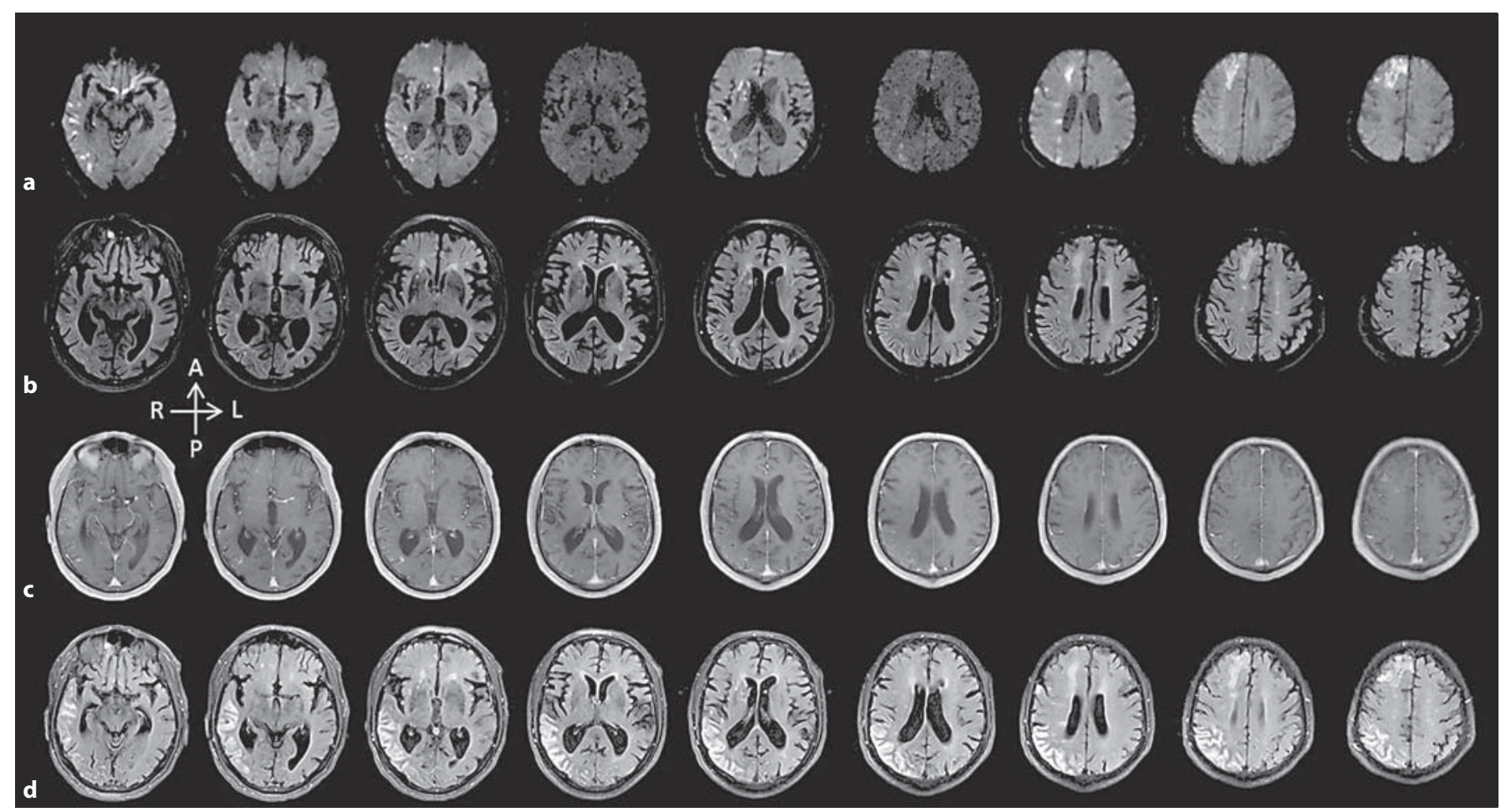

Fig. 1. MRI standard stroke protocol sequences and add-on FWC sequence. Standard sequences $(\mathbf{a}-\mathbf{c})$ revealed right frontal ischemic stroke with minute findings of right hemisphere watershed infarcts involving the right middle cerebral artery territory. a Diffusion-weighted imaging and apparent diffusion coefficient (not shown) sequences revealed restricted diffusion in the right frontal lobe with watershed distribution of restricted diffusion involving the right middle cerebral artery territory; findings consistent with a watershed ischemic stroke. A similar distribution of deficits was noted on the FLAIR sequence (b), supporting a subacute chronology for the event. c A $\mathrm{T}_{1}$-weighted sequence with contrast had a similar distribution of enhancement on initial evaluation. $\mathbf{d}$ The add-on FWC sequence revealed an extensive area of BBBD and BCSFD (HARM) over the right parietotemporal cortex, subregions of which also had cortical enhancement. In retrospect, parts of the same area could be considered slightly hyperintense on the FLAIR sequence and enhancing on the $\mathrm{T}_{1}$-weighted sequence with contrast. 